\title{
COBORDISM CLASSES OF SQUARES OF ORIENTABLE MANIFOLDS
}

BY PETER G. ANDERSON

\author{
Communicated by I. Singer, June 25, 1964
}

In this paper we give an outline of the following theorem. ${ }^{1}$ Full details will appear elsewhere.

THEOREM. If $M$ is an orientable manifold, then there exists a spin manifold $N$ such that $N$ is cobordant to $M \times M$ (in the unoriented sense). (For definitions and notation see [1] and [3].)

Following C. T. C. Wall [5] we construct a set of orientable manifolds whose cobordism classes generate the image of the "orientation

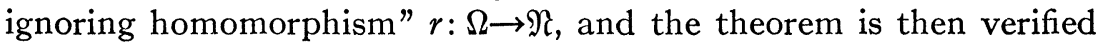
for each of these generators.

Some of these manifolds are certain complex projective spaces $C P^{n}$. As was noted in [2],CPn $\times C P^{n}$ is cobordant to quaternionic projective space $H P^{n}$. Since $H P^{n}$ is always 3-connected it is a spin manifold.

A second type of manifold used is constructed as follows. Let $\lambda$ be the canonical nontrivial line bundle over real projective space $P^{n}$, and $\epsilon^{m}$ the trivial $m$-plane bundle over $P^{n}$. Define $M(m, n)$ as the space of lines through the origin in each fibre of the Whitney-sum bundle $\lambda \oplus \epsilon^{n} . M(m, n)$ is an orientable manifold if and only if $m$ is odd and $n$ is even, and certain of these manifolds are used as generators for $r(\Omega)$.

The third type of manifold used is denoted by

$$
M\left(m_{1}, n_{1} ; m_{2}, n_{2} ; \cdots ; m_{r+1}, n_{r+1}\right),
$$

where $r \geqq 1, m_{i}$ is odd and $n_{i}$ is even for $i=1, \cdots, r+1$. This manifold is the total space of a certain fibre bundle over $S^{1} \times \cdots \times S^{1}(r$ factors), with fibre $M\left(m_{1}, n_{1}\right) \times \cdots \times M\left(m_{r+1}, n_{r+1}\right)$.

To prove the theorem for these last two types of manifolds we construct their "complex analogues" as follows. Let $c \lambda$ denote the canonical complex line-bundle over complex projective space $C P^{n}$, and $c \epsilon^{m}$ the trivial complex $m$-plane bundle over $C P^{n}$. Then $C M(m, n)$ is the space of complex lines through the origin in each fibre of $c \lambda \oplus c \epsilon^{m} . C M\left(m_{1}, n_{1} ; \cdots ; m_{r+1}, n_{r+1}\right)$ will be the total space of a fibre

${ }^{1}$ This theorem was originally conjectured by J. Milnor in [2]. 
bundle over $S^{2} \times \cdots \times S^{2}(r$ factors $)$ with fibre $C M\left(m_{1}, n_{1}\right) \times \cdots$ $\times C M\left(m_{r+1}, n_{r+1}\right)$.

If $M$ is one of our manifolds we use the following method to verify that $M \times M$ is cobordant to $C M . H^{*}\left(M ; Z_{2}\right)$ and $H^{*}\left(C M ; Z_{2}\right)$ are isomorphic truncated polynomial algebras over $Z_{2}$, the former on several one-dimensional generators and the latter on several twodimensional generators. If we represent this isomorphism by $D$ : $H^{*}(M) \rightarrow H^{*}(C M)$ (note that $D\left(H^{i}(M)\right)=H^{2 i}(C M)$ ), we may prove that $D$ preserves Stiefel-Whitney classes: $D(w(M))=w(C M)$. Since $w_{1}(M)=0, w_{1}(C M)=w_{2}(C M)=0$; hence $C M$ is a spin manifold.

Let $w_{i_{1}} \cdots w_{i_{k}}[M]$ denote a typical Stiefel-Whitney number of $M$; then by a theorem of Wall [5]

$$
w_{i_{1}} \cdots w_{i_{k}}[M]=w_{2 i_{1}} \cdots w_{2 i_{k}}[M \times M],
$$

and $w_{j_{1}} \cdots w_{j_{h}}[M \times M]=0$ if any of the $j$ 's is odd. Therefore

$$
w_{i_{1}} \cdots w_{i_{k}}[M \times M]=w_{i_{1}} \cdots w_{i_{k}}[C M]
$$

for any $i_{1}, \cdots, i_{k}$. So by $\mathrm{R}$. Thom's result [4], $M \times M$ is cobordant to $C M$.

\section{BiBLIOGRAPHY}

1. J. Milnor, Lectures on characteristic classes, Princeton University, Princeton, N. J., 1957.

2. - On the Stiefel-Whitney numbers of complex manifolds and of spin manifolds, Topology (to appear).

3. _- Spin structures on manifolds, Enseignement Math. 9 (1963), 198-203.

4. R. Thom, Quelques propriêtés globales des variêtés différentiables, Comment. Math. Helv. 28 (1954), 17-86.

5. C. T. C. Wall, Determination of the cobordism ring, Ann. of Math. (2) 72 (1960), 292-311.

Massachusetts Institute of Technology 